\title{
One-way fluid-solid coupling analysis of subsurface safety valve plate
}

\author{
Meiqiu Li ${ }^{1}$, Kang Yang ${ }^{1}$, Junfeng Zhao ${ }^{2}$, Jingbo Luo ${ }^{1}$, Ning $\mathrm{Li}^{1}$ \\ ${ }^{1}$ College of Mechanical Engineering, Yangtze University, Jingzhou 434023, China \\ ${ }^{2}$ Serva SJS Limited, Jingzhou 434000, China
}

Corresponding Author Email: limeiqiu@ sina.com

https://doi.org/10.18280/ijht.360207

Received: 30 September 2017

Accepted: 27 February 2018

\section{Keywords:}

subsurface safety valve, fluid-solid coupling, opening resistance, resistance torque

\begin{abstract}
When the working stratum pressure of the subsurface safety valve is large, the fluid in the well will have a greater impact on the valve plate, and even directly determine the opening resistance of the subsurface safety valve plate. By fluid analysis, we can get the fluid pressure cloud diagram and the velocity distribution cloud diagram of the valve plate at different opening angles, also we can get the opening resistance of the valve plate under the effect of the fluid, as well as the relationship between the resistance torque of the fluid resistance around the valve plate pinhole and the opening angle of the valve plate, providing certain guidance for the opening operation of the subsurface safety valve. Through the one-way fluid-solid coupling analysis, the stress cloud diagram and the maximum equivalent stress change under the effect of the fluid load are obtained when the valve plate is opened at different angles.
\end{abstract}

\section{INTRODUCTION}

The subsurface safety valve (SSSV) installed underground is an important device to ensure the safety of oil and gas production. It should be closed under abnormal operation to prevent blowout and other accidents. At present, oil and gas wells are developing toward deep wells and ultra-deep wells. Therefore, the installation depth of subsurface safety valves is also gradually deepening, and the pressure of the working environment of the subsurface safety valve will also change a lot. The high-pressure oil and gas liquid in the well will have a great effect on the opening of the valve plate.

While the subsurface safety valve is opening, as the highpressure oil-gas fluid flows through the valve plate and the valve seat, due to the presence of the valve plate and the change of the valve plate opening angle, it will severely block the fluids, and in turn the flow of the fluid will obstruct the opening of the valve plate, therefore, with the opening of the plate, the resistance of the fluid received by the valve plate during the opening process changes momentarily, and the opening resistance of the fluid to the valve plate at different opening angles varies greatly.

According to the on-site statistics, the subsurface safety valve often encounters difficulty in valve plate opening and jamming after the valve plate opens at a certain angle during the opening process. Therefore, analysis on the force acting on the upper and lower surface of the valve plate by the subsurface fluid during the opening of the valve plate, namely the fluid resistance of the valve plate and the resistance torque of the fluid resistance around the hinge pin of the valve plate, can instruct the wellhead pressure control system to adjust the pressure of the hydraulic control line so that the valve plate of the subsurface safety valve can be opened more smoothly and reliably. The failure probability of subsurface safety valve and the power consumption of hydraulic control are effectively reduced in the course of subsurface operation.

\section{FLUID ANALYSIS UNDER DIFFERENT OPENING ANGLES OF VALVE PLATE}

\subsection{Construction of runner model under different opening angles}

After the subsurface safety valve is opened, hole bottom fluid flows through the valve plate joint part to the inside of the valve body, and finally flows through the oil pipe to the ground recovery pipeline. At the valve seat and lower joint, due to the gradual opening of the valve plate, the fluid space and fluid state near the valve plate will change, and the changing fluid will have a greater effect on the valve plate on the valve seat, so the fluid simulation position is mainly for the fluid near the valve plate.

The opening process of subsurface safety valve plate is a continuous process, that is, the valve plate continuously rotates around the hinge pin of the valve plate. Generally, dynamic mesh analysis method can be used to simulate the fluid model with fluid boundary changes, considering the complexity of the structure of subsurface safety valve plate, and in dynamic mesh analysis, the process of boundary changing and mesh disappearing and rebuilding are very complicated, so in the simulation, it is very difficult to achieve rotation synchronization between the fluid and the valve plate. This study uses a relatively simple quasi-static state simulation analysis method to respectively stimulate and calculate eight instantaneous conditions of the valve plate when the opening angle is $10^{\circ}, 20^{\circ}, 30^{\circ}, 40^{\circ}, 50^{\circ}, 60^{\circ}, 70^{\circ}, 80^{\circ}$ respectively. The opening angel of subsurface safety valve is shown in Figure 1:

The nominal size of the subsurface safety valve is chosen to be $2 \frac{7}{8}$ inch. In the 3D software, the fluid domain model of the valve plate attachment can be obtained by Boolean calculation, after which the valve plate position has formed corresponding boundary conditions, the profile of subsurface safety valve plate fluid field is shown in Figure 2: 


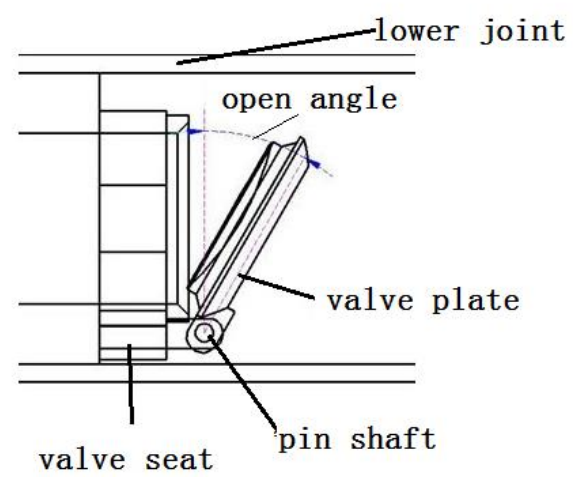

Figure 1. Opening angle of subsurface safety valve plate

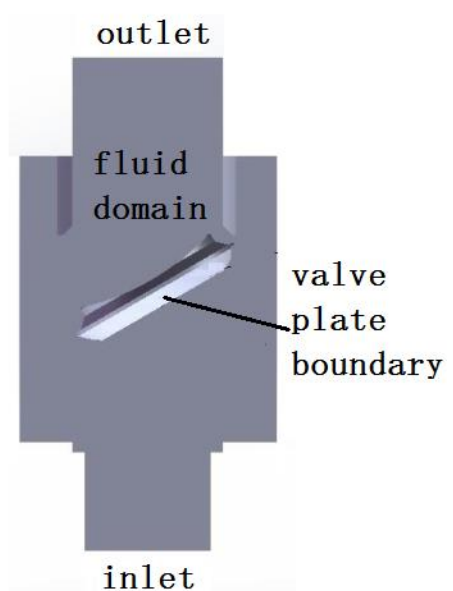

Figure 2. Subsurface safety valve fluid domain model

\subsection{Determination of meshing and boundary conditions}

The finite volume method is generally used in fluid simulation, it is also commonly used as a numerical algorithm. The finite volume method mainly uses numerical discrete [1]. Its theoretical basis is the conservation equation of the integral form, and the conservation formula is satisfied for each finite volume.

$$
\frac{\partial}{\partial t} \int_{\Omega} Q d \Omega+\int_{\Gamma} d \Gamma=0
$$

where Q is the target quantity; $\Omega$ is the finite volume; $\Gamma$ is the flow at a finite volume boundary $\Gamma$.

This equation describes the calculation of each control body defined by the mesh. The finite volume method [2-4] focuses on the construction of discrete equations from a physical point of view. Each discrete equation is a conservative representation of a certain physical quantity on a finite size volume. The physical concept of the derivation process is clear, and the discrete equation coefficients have certain physical significances, which ensure that the discrete equations have conserved properties [5-6].

The fluid performance parameters in FLUENT analysis have a great influence on the analysis results. In order to make the subsurface fluid materials conform to the research requirements, the properties of fluids in different reservoir environments can be obtained by referring to relevant literatures, that is, the density and viscosity of fluid under different stratum pressures, as shown in Table 1 .

Take a beach oil well as an example [7], combining with the data in Table 1, we can get the stratum pressure at the bottom of the valve plate is $30.0 \mathrm{MPa}$, the density of the fluid flowing through the valve plate is $0.7814 \mathrm{~g} / \mathrm{cm} 3$, and the hydrodynamic viscosity is $10.6 \mathrm{cp}$. For the subsurface safety valve with selfbalancing function, before the valve plate is opened, the pressure difference between the upper and lower surface of the valve plate is nearly balanced, set corresponding boundary conditions, the inlet pressure is the bottom pressure in the well $30 \mathrm{MPa}$. Since the pressure on upper part of the valve and the bottom hole pressure are approximately equal after the pressure of upper and lower surface of valve plate is equalized, there is still a certain distance between the outlet section and the upper surface of the valve. Considering the hydrostatic pressure and the pressure distribution on upper part of the valve, the outlet pressure is set to $28 \mathrm{MPa}$. Considering that the model under research is an internal basin, the standard k- $\varepsilon$ model is more suitable for the hydrodynamic analysis when the valve plate is opened because the obstruction of the valve plate will cause the valve body to exhibit high.

Table 1. Fluid parameters

\begin{tabular}{ccc}
\hline Pressure(MPa) & Density $\left(\mathbf{g} / \mathbf{c m}^{\mathbf{3}}\right)$ & Viscosity $(\mathbf{c p})$ \\
\hline 30.0 & 0.7814 & 10.6 \\
25.0 & 0.7766 & 9.516 \\
20.0 & 0.7721 & 8.564 \\
16.0 & 0.7690 & 8.044 \\
11.8 & 0.7632 & 7.902 \\
\hline
\end{tabular}

\subsection{Fluid pressure distribution cloud}

According to the analysis model established above, through FLUENT simulation we can know that [8-9], with the change of the opening angle of the valve plate, the fluid pressure near the valve plate presents a certain regularity, the fluid pressure cloud diagram of several opening angles $\alpha$ is shown in Figure 3:

Figure 3 shows that the pressure of the fluid near the cone sealing surface of the valve plate varies greatly at different opening angles of the valve plate, the fluid pressure $P$ near valve plate inflow side cone sealing surface has a relationship with the opening angle $\alpha$ as shown in Figure 4:

It can be seen from Figure 4 that when the valve plate opening angle is less than 10 degrees or more than 80 degrees, in the former situation, since the opening angle is small, fluid flows mainly from the edge of the valve plate; for the later situation, as the opening angle of valve plate is large, the main pressure surface is near the edge, which makes the fluid pressure near the edge of the inflow side of the valve plate larger in both situations. When the opening angle of the valve plate varies between 10 degrees to 80 degrees, the area near the valve plate cone sealing surface is the low-pressure area, and the fluid pressure shows a tendency of decreasing first and increasing later, when the valve plate opening angle is about 50 degrees, the sealing surface low-pressure area has a minimum pressure of about $27 \mathrm{MPa}$. 


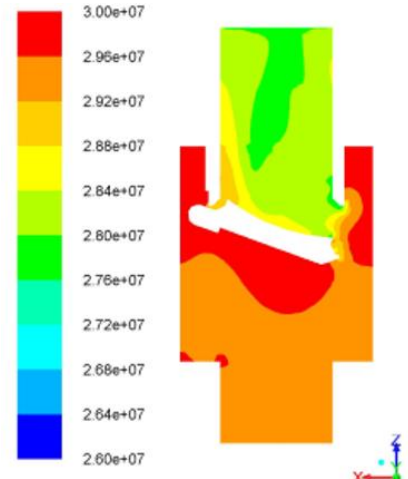

(a) $\alpha=20^{\circ}$

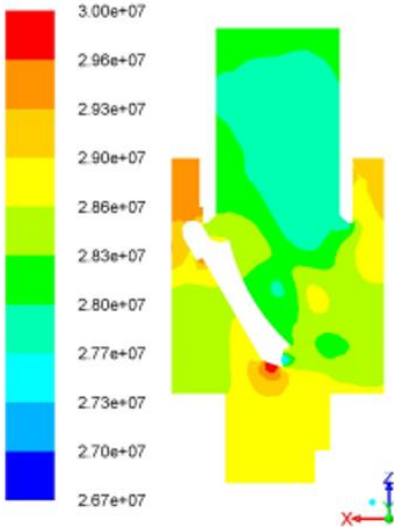

(c) $\alpha=60^{\circ}$

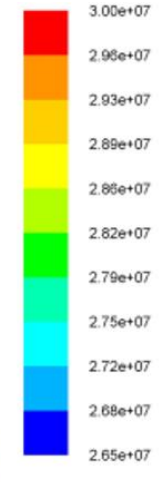

(b)
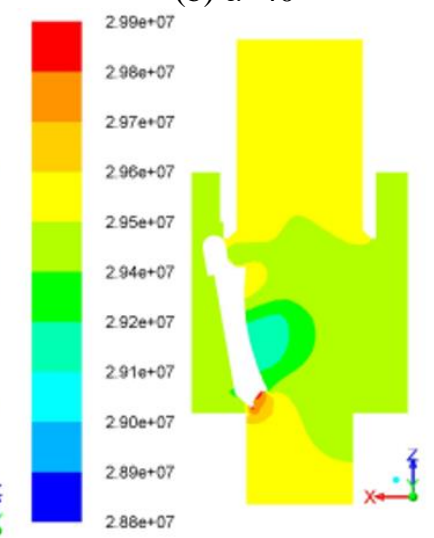

(d) $\alpha=80^{\circ}$
Figure 3. Fluid pressure cloud diagram of several opening angles

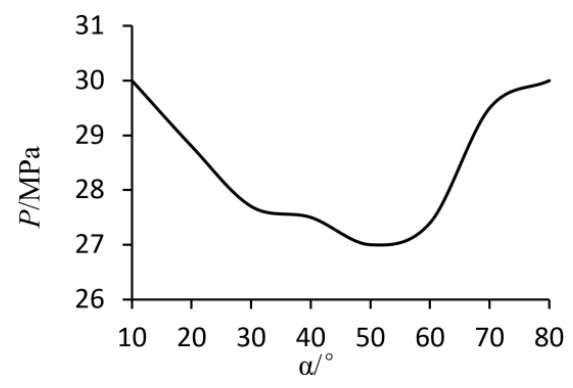

Figure 4. Relationship between fluid pressure of valve plate sealing surface and the opening angle

\subsection{Flow rate distribution cloud}

To establish the profile of the valve body, the rate distribution cloud diagrams of the fluid domain under several opening angles are shown in Figure 5:

Due to the existence of the valve plate, the fluid is prone to generate vortices where the structure changes abruptly, from the rate cloud diagram, it can be seen that when the valve plate opening angle changes from 0 degree to 60 degrees, the low speed vortex area away from the hinge pin side of the valve plate gradually decreases. When the valve plate opening angle is 60 degrees, the low speed vortex away from the hinge pin side of the valve plate basically disappears.

When the valve plate opening angle changes from 60 degrees to 80 degrees, the low speed vortex area away from the hinge pin side of the valve plate gradually becomes larger. When the opening angle is 60 degrees, in the near valve plate area on the outflow side of the valve plate, it will form fluid vortices, and the low speed vortex area gradually becomes larger as the opening angle increases.

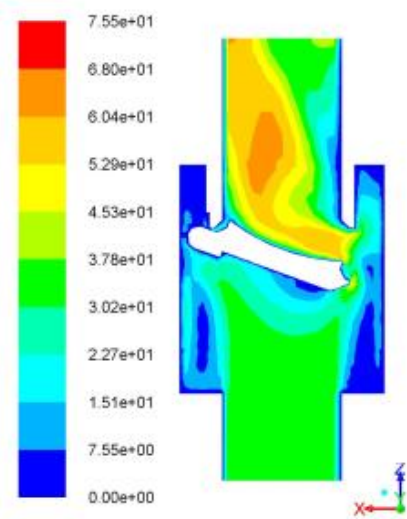

(a) $\alpha=20^{\circ}$

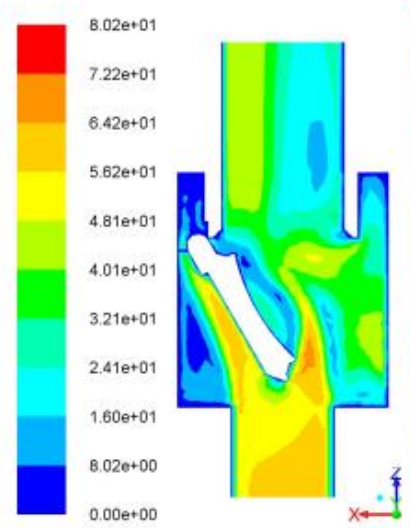

(c) $\alpha=60^{\circ}$

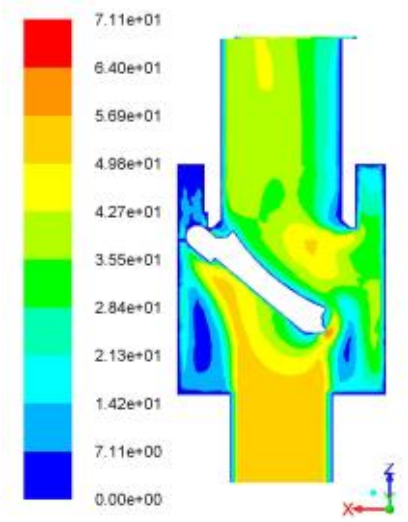

(b) $\alpha=40^{\circ}$
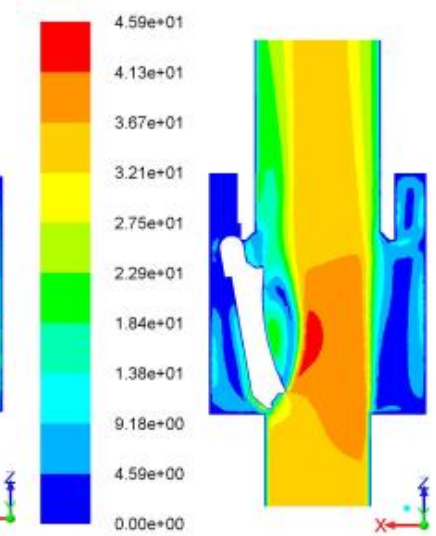

(d) $\alpha=80^{\circ}$
Figure 5. Flow rate distribution cloud diagram at different opening angles

\section{SUBSURFACE SAFETY VALVE PLATE OPENING RESISTANCE AND TORQUE}

\subsection{The force of fluid acting on the valve plate under the same opening angle}

Under different opening angles of the subsurface safety valve, due to changes in fluid pressure near the valve plate, there are large differences in fluid pressure distribution in different parts of the valve plate. The pressure distribution of the fluid has a great influence on the opening resistance of the subsurface safety valve plate, therefore, it is necessary to calculate the fluid resistance and the fluid resistance change trend along the axial direction of the subsuface safety valve under different opening angles.

Taking the opening angle of the valve plate at 30 degrees as an example, the forces acting on the upper and lower surfaces of the valve plate can be obtained as $3700.46 \mathrm{~N}, 3050.24 \mathrm{~N}$, respectively, and the resultant force is $650.22 \mathrm{~N}$. The direction of the force is opposite to the $\mathrm{Z}$ direction in the flow cloud diagram, namely the fluid hinders the opening of the valve plate. As the subsurface fluid medium is viscous, the viscous resistance is $0.46 \mathrm{~N}$ and $1.18 \mathrm{~N}$, respectively. Finally, the total force is $651.86 \mathrm{~N}$, and the force of the fluid obstructs the opening of the subsurface safety valve plate.

Changing the opening angle of the valve plate, the relationship between the resistance $F_{\mathrm{z}}$ and the opening angle $\theta$ 
is shown in Figure 6.

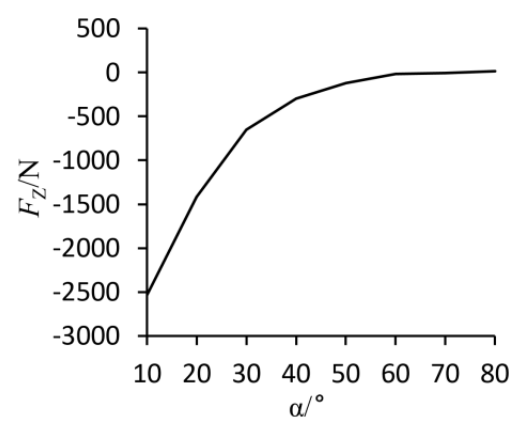

Figure 6. Relationship between fluid resistance and opening angle

Figure 6 shows that as the opening angle of the valve plate increases, the resultant force received by the valve plate gradually decreases, and when the valve plate opening angle is less than 70 degrees, the effect of the fluid on the valve plate is an impediment, when the valve plate opening angle is greater than 70 degrees, the effect becomes a pushing power, that is, the flow of fluid facilitates the opening of the subsurface safety valve plate.

\subsection{Torque of fluid to valve plate at different opening angles}

When the subsurface safety valve is opening, the valve plate rotates around the valve plate hinge pin, set corresponding rotation center and rotation axis, according to the relevant parameters when the fluid model is established, we can get the coordinates of the rotation center of the valve plate as $(0.051$, $0,-0.3268)$. Since the hinge pin is mounted on the valve seat along the $\mathrm{Y}$ direction, the rotation axis of the valve plate is $(0,1,0)$.

By changing the opening angle of the valve plate in the model, we can get the relationship between the torque $M_{\mathrm{Z}}$ of fluid to the valve plate and the opening angle as shown in Figure 7:

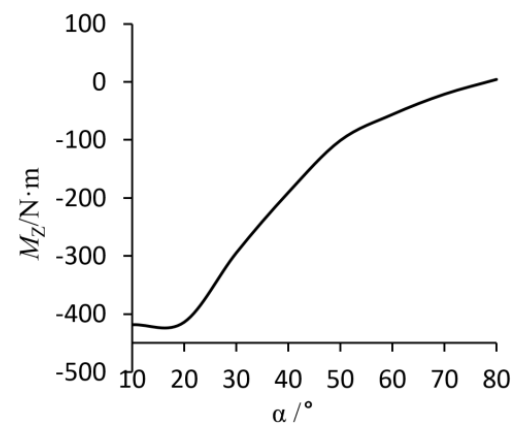

Figure 7. Relationship between fluid resistance torque and opening angle

Figure 7 shows that as the opening angle of the valve plate increases, the torque of fluid to the valve plate is gradually reduced and then increases in the opposite direction. When the opening angle of the subsurface safety valve is less than 70 degrees, the effect of the fluid on the opening of valve plate is an impediment, when the valve plate opening angle is greater than 70 degree, the fluid has a pushing power torque to the opening of the valve plate.

\section{ONE-WAY FLUID-SOLID COUPLING ANALYSIS OF SUBSURFACE SAFETY VALVE}

When the valve plate of the subsurface safety valve is opening, along with the change of the opening angle of the valve plate, on the one hand, the valve plate will hinder the flow of fluid inside the subsurface safety valve; on the other hand, due to the presence of high-speed flowing fluid near the valve plate, and the fluid pressures on the upper and lower surfaces of the valve plate are different, that is, the fluid will transmit the fluid pressure to the valve plate, and the fluid will have a certain influence on the solid structure, especially the valve plate area.

In order to obtain the effects of fluid on solids, fluid-solid coupling calculations can be used. Fluid-solid coupling calculations can be divided into two categories according to the data trans-mission methods: one-way coupling and twoway coupling. One-way coupling mainly refers to the transfer of fluid computation data to the solid, which typically transfers the fluid pressure or only moves from the solid computational transmission mesh node to the fluid. After one-way coupling is applied to the effect of the flow field on solids, the deformation of the solid is not significant, that is, the boundary morphology of the flow field changed little, which does not affect the smooth distribution, and the subsurface safety valve is fit for one-way fluid-solid coupling analysis.

\subsection{The construction of one-way fluid-solid coupling model}

Through the corresponding modeling and assembly, the fluid-solid coupling model of the fluid domain and solid boundary of the subsurface safety valve can be obtained, as shown in Figure 8:

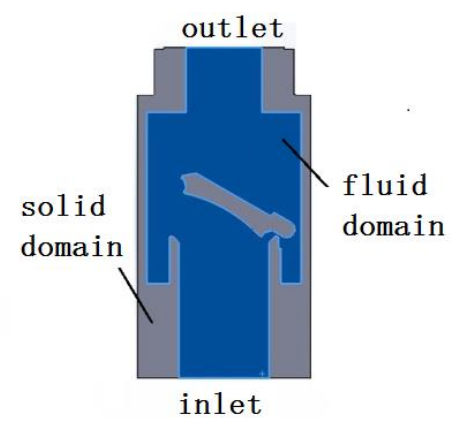

Figure 8. Fluid-solid coupling model of subsurface safety valve

Using the one-way fluid-solid coupling analysis module in Workbench, as in the one-way flu-id-solid coupling analysis, the data is transmitted in single direction, the results of fluid analysis will be transmitted as the load of solid analysis [10].

\subsection{Selection of load surface and transmission of fluid load}

In the fluid analysis, the surface between the fluid domain and the solid surface is named in-terface, select the interface surface in the fluid model, and the results of fluid analysis are loaded into the solid model in the form of pressure[11-13]. The load model is shown in Figure 9.

Figure 9 shows the situation where the fluid pressure load spectrum acts on the solid surface when the valve plate opening angle is 30 degrees. The entire inner surface of the valve body is affected by the pressure of the fluid, and the 
loading pressure of the inflow side of valve plate is larger than that of the outflow side of the valve plate, and the difference in the pressure of upper and lower surface of the valve plate is close to $3 \mathrm{MPa}$.

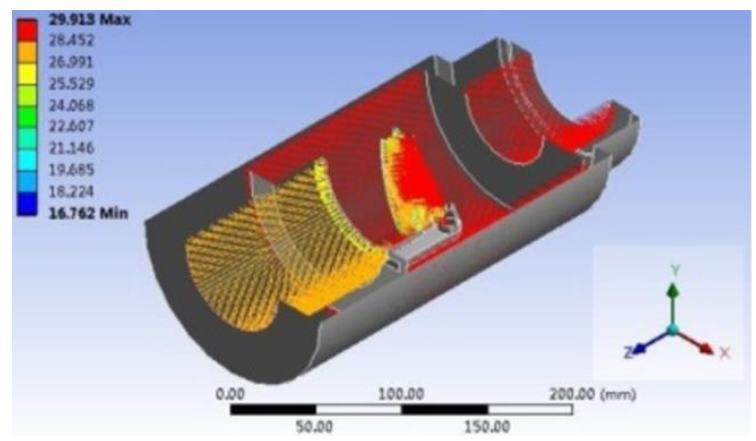

Figure 9. Load model

In the solid model simulation, the fluid model is suppressed, set the valve plate pinhole and the hinge pin as a friction contact pair, set the friction coefficient to 0.1 .

\subsection{Fluid-solid coupling analysis results}

According to the method of elastic mechanics, the pinholes of the valve plate and the inner and outer surfaces of the valve seat hinge pin are in contact with each other. When the contact position is subjected to pressure, the contact surface will occur elastic deformation. At this time, the contact position will change from line contact to surface contact, and the contact surface can be expanded into a rectangular contact surface to generate contact stress at the contact surface, the model is shown in Figure 10

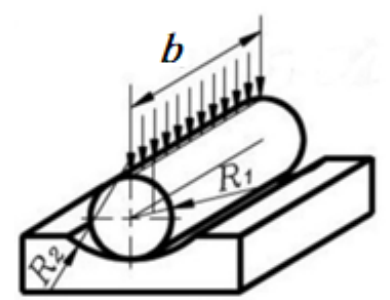

Figure 10. Contact model of hinge pin and the pinhole

The calculation of the theoretical contact stress of the model is in accordance with the Hertz formula. When the valve seat hinge pin is coupled with the pinholes of the valve plate, the theo-retical contact stress formula is [14]:

$$
\sigma_{\max }=\sqrt{\frac{F}{\pi b}\left(\frac{\frac{1}{R_{1}}-\frac{1}{R_{2}}}{\frac{1-\mu_{1}^{2}}{E_{1}}+\frac{1-\mu_{2}^{2}}{E_{2}}}\right)}
$$

In the formula, $F$ is the normal pressure between contact surfaces $(\mathrm{N})$; $\mathrm{b}$ is the length of contact $(\mathrm{m}) ; R_{1}$ is the hinge pin radius (m); $R_{2}$ is the valve plate pinhole radius (m); $E_{1}, E_{2}$ are the elastic modulus (MPa); $\mu_{1}, \mu_{2}$ are the poisson's ratio.

The material of the valve seat hinge pin and valve plate is nichrome [15]. The elastic modulus of the material is $192 \mathrm{GPa}$, the Poisson's ratio is 0.27 , and the yield limit of the material is $1100 \mathrm{MPa}$. According to SY/T 10024-1998, when the subsurface safety valve type is $3^{1} / 2$ inch, the diameter of the pinhole of the valve plate is $8.5 \mathrm{~mm}$, the diameter of the hinge pin is $8 \mathrm{~mm}$, and the contact length between the valve plate pinhole and the valve seat hinge pin is $9 \mathrm{~mm}$. Substituting corresponding data to simplify formula (2) we can get:

$$
\sigma_{\text {max }}=\sqrt{\frac{F E}{2 \pi b\left(1-\mu^{2}\right)} \cdot \frac{R_{2}-R_{1}}{R_{1} R_{2}}}
$$

Refer to the axial force of the fluid resistance of the subsurface safety valve plate under different opening angles, select $\mathrm{F}=2530 \mathrm{~N}$ as the normal pressure of the contact surface between the valve plate pinhole and the valve seat hinge pin, substitute into formula (3) we can get the contact stress between the valve plate pinhole and the valve seat hinge pin as $\sigma=369 \mathrm{MPa}$.

Taking the opening angle of the valve plate of the subsurface safety valve at 30 degrees as an example, through the one-way fluid-solid coupling analysis, when the inlet pressure is $30 \mathrm{MPa}$ and the outlet pressure is $28 \mathrm{MPa}$, the stress cloud diagram of the valve plate and the valve seat under the effect of fluid pressure can be obtained as Figure 11:

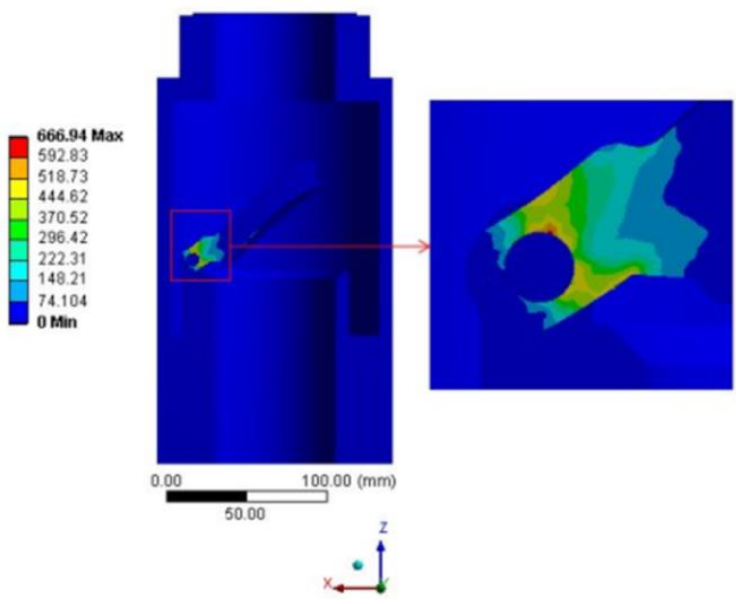

Figure 11. Fluid-solid coupling stress cloud diagram with valve plate opening angle at 30 de-grees

Figure 11 shows that the maximum equivalent stress of the solid model under the effect of the fluid load appears at the place where the valve plate pinhole and the valve seat hinge pin contact, and the maximum equivalent stress is $666.94 \mathrm{MPa}$, the maximum equivalent stress is less than the yield limit of the material $1100 \mathrm{MPa}$, which meets the strength requirement.

Changing the opening angle of the valve plate we can obtain the relationship between the maximum stress Pm and the opening angle $\alpha$ of the valve plate at different opening angles, as shown in Figure 12.

From the results of fluid-solid coupling analysis, it can be seen that the maximum equivalent stress of the subsurface safety valve occurs at the position where the pinhole of the valve plate and the valve seat hinge pin contact, with the increase of the opening angle of the subsurface safety valve, the maximum equivalent stress of the pinhole of the valve plate is gradually reduced. When the valve plate opening angle is 10 degrees, the stress of the valve plate pinhole is the 
maximum, and the maximum value is $852.47 \mathrm{MPa}$, which is less than the yield limit of the material.

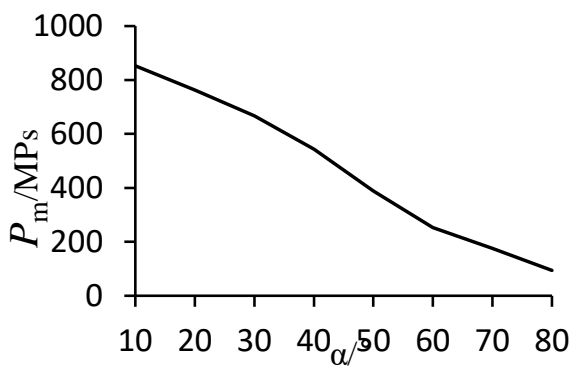

Figure 12. Maximum equivalent stress of fluid-solid coupling analysis at different opening angles

\section{CONCLUSION}

(1) For a certain type of subsurface safety valve, through the fluid simulation, we have obtained the valve body fluid pressure distribution cloud diagram at different valve opening angles, and the variation of the maximum fluid pressure at the inflow side of the valve plate.

(2) By corresponding post-processing of the fluid analysis results, we have obtained the relationship between the resistance of the well fluid to the valve plate and the opening angle of the valve plate; the relationship between the fluid resistance torque around the pinhole of the valve plate and the opening angle of the valve plate. All these provide certain guidance for the ground pressure control system when the subsurface safety valve plate opens.

(3) By establishing the fluid-solid coupling model under different opening angles and adopting one-way fluid-solid coupling analysis, we have obtained the stress cloud diagram of the solid model under the effect of fluid load when the valve plate of the subsurface safety valve is opened at different angles, as well as the relationship between the maximum stress and opening angle.

\section{ACKNOWLEDGEMENTS}

The projects supported National Key R \& D project (2016YFC0303703) and Hubei Province Natural Science Foundation(2016CFC740) are gratefully acknowledged.

\section{REFERENCES}

[1] Corvaro F, Nardini G, Paroncini M, Vitali R. (2015). Pi $\mathrm{v}$ and numerical analysis of natural convective heat trans fer and fluid flow in a square cavity with twovertical obs tacles. International Journal of Heat and Technology 33( 2): 51-56. https://doi:10.18280/ijht.330208

[2] Diaz JJDC, Nieto PJG, Mere JO, Garcia AB. (2007). Computer simulation of the laminar nozzle flow of a nonNewtonian fluid in a rubber extrusion process by the finite volume method and experimental comparison. Journal of Crystalline Solids 353(8-10): 981-983. https://doi.org/10.1016/j.jnoncrysol.2006.12.067
[3] Frigaard I, Vinay G, Wachs A. (2007). Compressible displacement of waxy crude oils in long pipeline startup flows, Journal of Non-Newtonian Fluid Mechanics 147(1): 45-64. https://DOI:10.1016/j.jnnfm.2007.07.002

[4] Zander V, Nitsche W. (2013). Control of secondary flow structures on a highly loaded compressor cascade,P.I. Mech. Eng. A-J. Pow. Energ 227(6): 674-682. https://DOI: 10.1177/0957650913495538

[5] Dossena V, Franchina N, Savini M, Marinoni F, Cecchi F, Bassi F. (2017). Reynolds number effects on the performance of safety valves operating with incompressible flows. Journal of Loss Prevention in the Process Industries(49): 525-535. https://doi.org/10.1016/j.jlp.2017.05.020

[6] Amarasinghe WS, Jayarathna CK, Ahangama BS, Moldestad BME, Tokheim LA. (2017). Experimental study and CFD modelling of minimum fluidization velocity for geldart A, B and D particles. International Journal of Modeling \& Optimization, 7(3): 152-156. https://doi.org/10.7763/IJMO.2017.V7.575

[7] Li MQ, Yang K, Zhou SZ, Zhang S. (2017). Dynamic equilibrium response analysis for self-balancing subsurface safety valve. Chinese Hydraulics and Pneumatics (6): 70-74. https://doi:10.11832/j.issn.10004858.2017.06.014

[8] Pendyala R, Wong YS, Ilyas SU. (2015). CFD simulations of natural convection heat transfer in enclosures with varying aspect ratios. Chemical $\begin{array}{lll}\text { Engineering } & \text { Transactions } & \text { 45(9): } 1348 .\end{array}$ http://doi:10.3303/CET1545133

[9] Pendyala R, Jia LC, Ilyas SU. (2015). CFD analysis of heat transfer performance in a car radiator with nanofluids as coolants. Chemical Engineering Transactions 45: 1261-1266. http://doi:10.3303/CET1545211

[10] Žižka M, Šulc R, Ditl P. (2017). Heat transfer between gas and liquid in a bubble column. Chemical Engineering Transactions 57(211): http://doi:10.3303/CET1757211

[11] Snider DM. (2001). An incompressible three dimensional multiphase particle-in-cell model for dense particle flows. Journal of computational physics 170(2): 523-549. https://doi.org/10.1006/jcph.2001.6747

[12] Gbadebo SA, Cumpsty NA, Hynes TP. (2007). Control of three-dimensional separations in axial compressors by tailored boundary layer suction, ASME.J. Turbomach, 130(1): 011004-011004-8. https://DOI: $10.1115 / 1.2749294$

[13] Chen F, Chen H. (2013). A BrokenP1-Nonconforming Finite Element Method for Incompressible Miscible Displacement Problem in Porous Media, ISRN Applied Mathematics 2013: 1-7. https://DOI: $10.1155 / 2013 / 498383$

[14] Li Y. (2015). Research on calculation of pin mechanics about hydraulic support. Coal Mine Machinery 36(10): 19-21. http://doi:10.13436/j.mkjx.201510008

[15] Li YS, Dong SX. (2017). Finite element aanalysis on flow field and stress field of SSSV flapper open/close. Drilling and Production Technology 40(1): 61-64. http://doi:10.3969/J.ISSN.1006-768X.2017.01.17 